www.jmscr.igmpublication.org

Impact Factor 5.84

Index Copernicus Value: 83.27

ISSN (e)-2347-176x ISSN (p) 2455-0450

crossref DOI: _https://dx.doi.org/10.18535/jmscr/v5i5.66

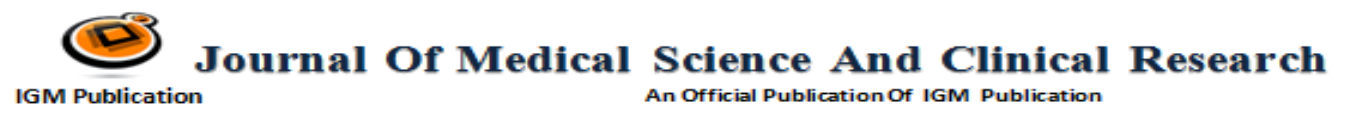

An official Publication of IGM Publication

\title{
Period Prevalence of Bronchiectasis in Moderate and Severe COPD
}

\author{
Authors \\ Dr Gunaseelan.G ${ }^{1}$, Dr Jayaprakash.B ${ }^{2}$, Dr Roy. $\mathbf{N}^{3}$ \\ ${ }^{1}$ Senior Resident, ${ }^{2}$ Additional Professor, Department of Pulmonary Medicine, ${ }^{3}$ Professor \& Head of \\ Radiodiagnosis. Govt. Medical College, Trivandrum, Kerala, India \\ Corresponding Author \\ Dr Jayaprakash.B
}

Additional Professor, Dept. of Pulmonary medicine, Govt. Medical College, Trivandrum, Kerala.695011

Tel: 9447658148; Email: jayansindhu@yahoo.com

\section{Abstract}

Chronic obstructive pulmonary disease (COPD), a common preventable and treatable disease characterised by persistent airflow limitation that is usually progressive and associated with an enhanced chronic inflammatory response in the airways and the lung to noxious particles or gases. Exacerbations and comorbidities contribute to overall severity in individual patients. Many studies have observed an association between the COPD and bronchiectasis, reporting the presence of bronchiectasis in up to 50\% of patients with moderate to severe COPD, this suggests that there may be a causal relationship in which COPD is a risk factor for bronchiectasis. Aim of the study: To find the prevalence of bronchiectasis in association with severe and moderate COPD admitted in Respiratory medicine department, Trivandrum over a period of 2 years

Secondary Objective: Relating dyspnoea scale (MMRC), FEV1, amount of daily sputum production $(<10$ $\mathrm{ml}, 10 \mathrm{ml}-30 \mathrm{ml},>30 \mathrm{ml}$ ) to bronchiectasis.

Study design: Hospital based cross sectional study.

Study Period: January 2012-November 2013.

Study Population: Patients admitted to Respiratory medicine department between the study period who are diagnosed as COPD and labelled as severe and moderate COPD according to GOLD guidelines.

Moderate FEV1 50\% $\leq$ FEV1 < 80\% predicted. Severe FEV1 $<50 \%$ Predicted.

Exclusion criteria: Other causes such as patients with old history of tuberculosis.

Patient not fit to undergo HRCT as suggested by radiologist. Patient not willing to participate.

Methodology: Patient diagnosed as severe or moderate COPD who were willing to participate in the study underwent HRCT from our radiodiagnosis department. Also includes a questionnaire regarding exacerbations, smoking index, dyspnoea scale (MMRC), current smoking status, sputum culture if admitted for infective exacerbations. HRCT was read independently by a radiologist and chest physician with control blinding. If both agree then it will be taken into account. We also try to prove an association between various factors studied through questionnaire and bronchiectasis.

Results \& Conclusion: The period prevalence of bronchiectasis in patients with moderate and severe COPD was 34.7\%.MMRC (grading), FEV1, years of COPD had significant correlation with presence of bronchiectasis in moderate and severe COPD. Right lower lobe was the most common site. Cylindrical bronchiectasis was the most common type.

Keywords: COPD, Bronchiectasis, Prevalence. 


\section{Introduction}

Chronic obstructive pulmonary disease (COPD), a common preventable and treatable disease characterised by persistent airflow limitation that is usually progressive and associated with an enhanced chronic inflammatory response in the airways and the lung to noxious particles or gases. Exacerbations and comorbidities contribute to overall severity in individual patients ${ }^{(1)}$.An association between COPD and bronchiectasis has been observed in many studies, reporting the presence of bronchiectasis in up to $50 \%$ of patients with moderate to severe COPD. This suggest that there may be a causal relationship in which COPD is a risk factor for bronchiectasis ${ }^{(2,3)}$. Bronchiectasis and COPD share many characteristics and have common pathophysiology, clinical and functional view points ${ }^{(4)}$.

The relationship between COPD and bronchiectasis can be summarised as they are both common conditions especially in older patients ${ }^{(5,6)}$. Hence a study was planned to find out the prevalence of bronchiectasis in patients with moderate and severe COPD and also to find out the correlation between various factors such as sex, smoking index, FEV1\%, MMRC grading ,years of COPD and amount of sputum production during exacerbation in patients with moderate and severe COPD.

\section{Aim of Study}

To find out the prevalence of bronchiectasis in association with severe and moderate COPD admitted in Respiratory medicine department, Trivandrum over a period of 2 years.

Secondary objective: Relating dyspnoea scale (MMRC), FEV1, amount of daily sputum production $(<10 \mathrm{ml}, 10 \mathrm{ml}-30 \mathrm{ml},>30 \mathrm{ml})$ to bronchiectasis.

\section{Study design}

Hospital based cross sectional study

\section{Study population}

Patients diagnosed as COPD and spirometry showing moderate (FEV1 between 50\% and 80\%) and severe (FEV1 less than 50\%).

\section{Sample size}

$\mathrm{N}=4 \mathrm{pq} / \mathrm{l}^{*} 1$

$\mathrm{P}$ - Prevalence calculated from a study published in chest journal

$\mathrm{Q}=100-\mathrm{p}$

$\mathrm{I}=\mathrm{p} / 5$

$\mathrm{N}=4(57.6)(42.4) / 11.52(11.52)$

$\mathrm{N}=73.66(74)$

\section{Exclusion criteria}

1. Patients with other causes for bronchiectasis

2. Patients not fit to undergo HRCT as suggested by radiologist

3. Patients not willing to give consent

\section{Methodology}

Study tool: structured questionnaire and HRCT

Patients diagnosed as severe and moderate COPD as per GOLD guidelines who are willing to participate in the study were examined using a structured questionnaire regarding smoking index, dyspnoea scale, exacerbations, current smoking status, sputum culture etc. They underwent HRCT from radiodiagnosis department. HRCT was read by a radiologist and pulmonologist with control blinding. If both agree then it will be taken into account.

\section{Results}

138 patients diagnosed as moderate and severe COPD based on clinical and spirometry underwent HRCT from radiodiagnosis department. 48 (34.7\%) patients out of 138 moderate and severe COPD had bronchiectasis (Fig 1).119(86.2\%) were males and $19(13.8 \%)$ were females.40 males and 8 females had bronchiectasis. There was no significant correlation $(p$ value $=0.470$ ) between sex of the patient and bronchiectasis in moderate and severe COPD. When FEV1 was less than $30 \%, 27$ patients had bronchiectasis and 5 had no bronchiectasis .With FEV1 between 30 and 50\%, 21 patients had bronchiectasis and 79 had no bronchiectasis. No patients showed bronchiectasis when FEV1 was between 50 and 70\% (Table.1). 


\section{JMSCR Vol||05||Issue||05||Page 21634-21638||May}

MMRC grading and presence of bronchiectasis was tabulated in table 2. The duration of COPD was graded in to mild(<5years), moderate $\quad 5-10$ years) and severe (>10years) and was correlated with the presence of bronchiectasis (Table.3). In our study there was no significant correlation between sex of the patient, amount of sputum production, smoking index and presence of bronchiectasis in moderate and severe COPD. HRCT showed lower lobe bronchiectasis in 34 (70.83\%) patients and right lower lobe was the most commonly involved lobe, 21 (43.75\%) (Fig.2). Cylindrical bronchiectasis was the most common type in our study which was seen in 33 $(68.75 \%)$, cystic in $11(22.91 \%)$ and varicose in 4(8.33\%) (Fig.3).

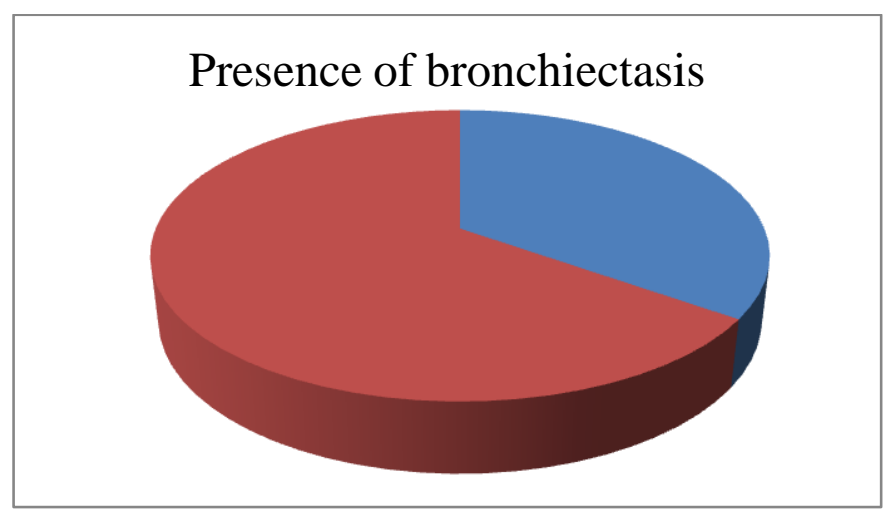

Fig 1: Bronchiectasis present in 37.3\%

Table 1: FEV1 and association with Bronchiectasis

\begin{tabular}{|l|c|c|c|c|}
\hline FEV1 & Less than 30 & $30-50$ & $50-70$ & P -value \\
\hline Bronchiectasis YES & 27 & 21 & 0 & 0.0001 \\
\hline Bronchiectasis NO & 5 & 79 & 6 & \\
\hline Total & 32 & 100 & 6 & \\
\hline
\end{tabular}

Table 2: Correlation between MMRC grading and Bronchiectasis ( $\mathrm{p}$-value 0.0003)

\begin{tabular}{|l|c|c|c|c|}
\hline MMRC Grading & 1 & 2 & 3 & 3 \\
\hline Bronchiectasis YES & 0 & 19 & 27 & 2 \\
\hline Bronchiectasis NO & 8 & 46 & 30 & 6 \\
\hline Total & 8 & 65 & 57 & 8 \\
\hline
\end{tabular}

Table 3: Years of COPD and bronchiectasis ( $p$-value 0.001)

\begin{tabular}{|l|c|c|c|}
\hline Years of COPD & $<5$ & $5-10$ & $>10$ \\
\hline Bronchiectasis YES & 2 & 23 & 23 \\
\hline Bronchiectasis NO & 13 & 60 & 17 \\
\hline TOTAL & 15 & 83 & 40 \\
\hline
\end{tabular}

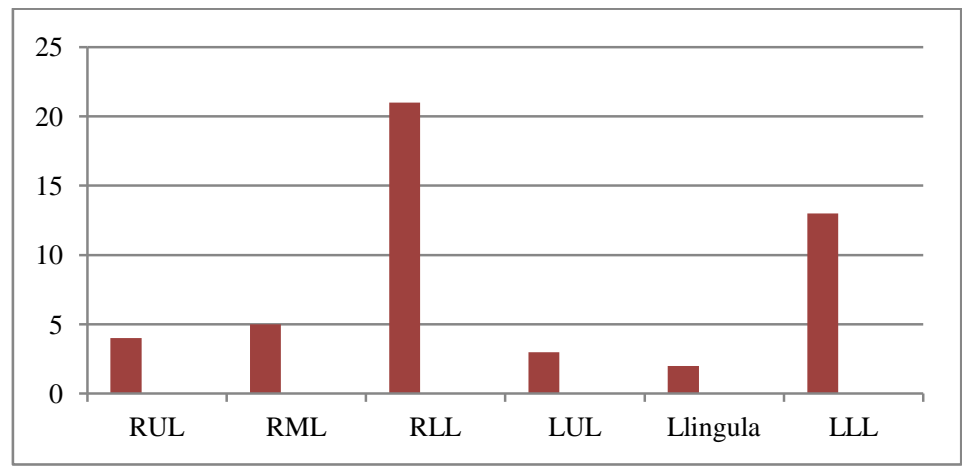

Fig 2: lobe wise distribution of Bronchiectasis in COPD 


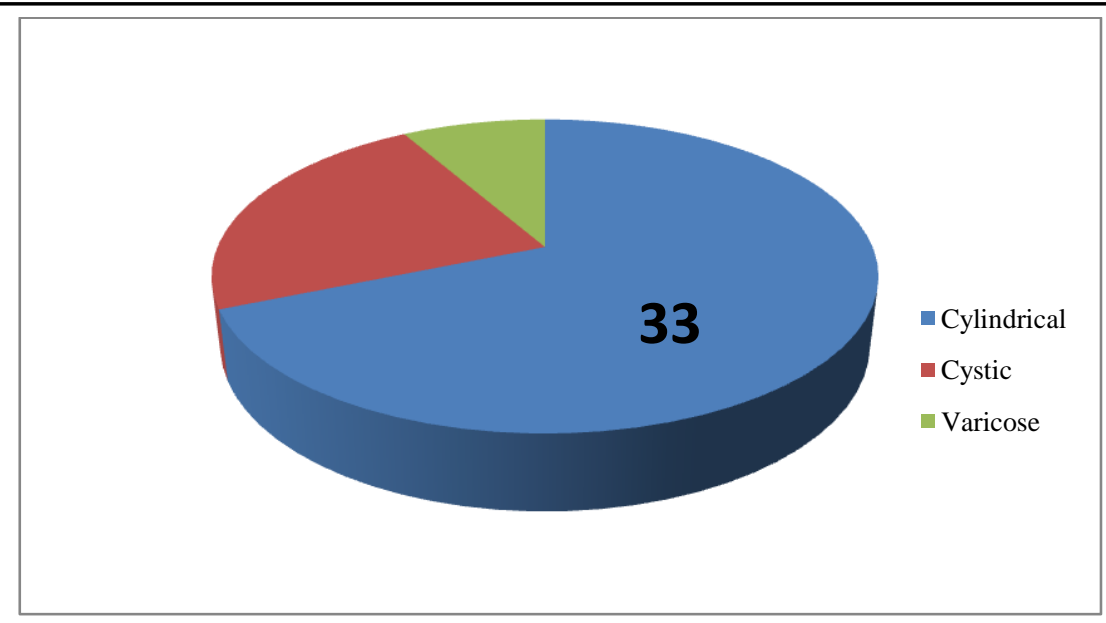

Fig 3: Type of Bronchiectasis in COPD

\section{Discussion}

COPD is diagnosed on the basis of poorly reversible airflow obstruction and is therefore a physiological diagnosis. Bronchiectasis is diagnosed in the presence of airway dilatation and airway wall thickening on imaging usually in HRCT and is therefore a structural diagnosis ${ }^{(7)}$. An increasing number of patients with COPD have a CT scan as part of their diagnosis and follow-up care, with consequent impact on the detection of airway wall changes. Physiological criteria for the diagnosis of COPD and structural criteria for the diagnosis of bronchiectasis create the possibility for individual patients to fulfil both, resulting conceptually in either co-diagnosis or an overlap syndrome between the two conditions. The prevalence of this overlap will vary depending on the respective prevalence of COPD and bronchiectasis in the population under consideration.

The period prevalence of bronchiectasis in moderate and severe COPD is $34.7 \%$ in our study.In a study published in CHEST journal the prevalence of bronchiectasis in moderate and severe COPD is $57.2 \%{ }^{(8)}$.Patel and colleagues had found a prevalence of bronchiectasis in $50 \%{ }^{(2)}$. Kessler $\mathrm{R}$ etal in their study showed a prevalence of $29 \%{ }^{(9)}$. Lower lobe bronchiectasis was the most common site in our study which was concordant with the above studies. Cylindrical bronchiectasis was the commonest type in both the studies and in our study also. Right lower lobe was the most common lobe involved in moderate and severe COPD in our study. Generally the commonest site for bronchiectasis is left lower lobe and the reason for right lower lobe predominance could not be established. Further studies are needed to find out the cause for right lower lobe predominance. We found a significant correlation between MMRC grading, number of years of COPD, FEV1 values and presence of bronchiectasis in moderate and severe COPD.

COPD and bronchiectasis share common symptoms of cough with sputum production and susceptibility to recurrent exacerbations driven by new or persistent infection. Patients presenting de novo may therefore result in a diagnostic challenge $^{(10)}$. The overlap between COPD and bronchiectasis is a neglected area of research and it is not covered by guidelines for clinical practice. Understanding the presence of bronchiectasis in COPD is important for future intervention and preventing disease progression ${ }^{(11)}$. Study done by Goeminne PC et al demonstrated a near threefold increased mortality rate, with patients with bronchiectasis and associated COPD having a 5year mortality of $55 \%$, compared with $20 \%$ in those with bronchiectasis without COPD ${ }^{(12)}$.

\section{Conclusion}

1) The period prevalence of bronchiectasis in patients with moderate and severe COPD was $34.7 \%$ 
2) Right lower lobe was the common site of bronchiectasis in patients with moderate and severe COPD.

3) Higher MMRC grading, lowFEV1, higher numbers of years of COPD are the risk factors for developing bronchiectasis in moderate and severe COPD.

\section{References}

1. Gold report 2013 available at www.goldcopd.org.

2. Patel IS, Vlahos I,Wilinson TMA. Bronchiectasis, exacerbation indices and inflammation in Chronic obstructive Pulmonary diseases. Am J Respir Crit Care Med.2004:170(4) 400- 07.

3. O'Brien C O,Guest PJ,Hill SL,Stokley RA .Physiological and radiological characterization of patients diagnosed with chronic obstructive pulmonary diseases in primary care.Thorax 2000;55(8):635-42

4. Stokley $R$ A. Neutrophils of COPD .Chest.2002;121 (sppl 5): 151S -155S

5. Pefia VS,Miravitlles M,Gabriel R. Geographic variations in prevalence and underdiagnosis of COPD:results of the IBERPOC multicentre epidemiological study.Chest.2000;118(4):981-89.

6. Weycker D,Edelsberg J,Oster G.Tino G.Prevalence and economic burden of Bronchiectasis .Cli Pulm Med.2005; 12(4):205-09

7. Pasteur MC, Bilton D, Hill AT.. British Thoracic Society guideline for non-CF bronchiectasis. Thorax 2010;65: Suppl. 1, i 1-i58.

8. Martínez-García MÁ ${ }^{1}$, Soler-Cataluña JJ, Donat Sanz Y, Catalán Serra P, Agramunt Lerma M, Ballestín Vicente J, PerpiñáTordera M. Factors associated with bronchiectasis in patients with COPD. Chest. 2011 Nov;140(5):1130-7.
9. Kessler R,Partridge MR,Miravitlles M,Cazzola M,Vogelmeier C,Leylnaud D et al.Symptom variability in patients with severe COPD: a pan-Eurpean cross sectional study. Eur Respir J 2011;37:264-72.

10. Hurst JR, Elborn JS, Soyza AD.COPD Bronchiectasis overlap syndrome. European Respiratory Journal2015 45:310-313.

11. Du Q, Jin J, Liu X, Sun Y. Bronchiectasis as a Comorbidity of Chronic Obstructive Pulmonary Disease: A Systematic Review and Meta-Analysis. Sethi S, ed. PLoS ONE. 2016;11(3):e0150532. doi:10.1371/journal.pone.0150532.

12. Goeminne PC, Nawrot TS, Ruttens D, et al. Mortality in non-cystic fibrosis bronchiectasis: a prospective cohort analysis. Respir Med 2014; 108: 287-296. 